\title{
Correction to: MicroRNA-34 Contributes to the Stress-related \\ Behavior and Affects 5-HT Prefrontal/GABA Amygdalar System through Regulation of Corticotropin-releasing Factor Receptor 1
}

\section{Diego Andolina ${ }^{1,2} \cdot$ Matteo Di Segni ${ }^{1,2}$. Alessandra Accoto ${ }^{1,2} \cdot$ Luisa Lo lacono ${ }^{1,2}$. Antonella Borreca ${ }^{2,3}$. Donald lelpo $^{2}$ - Nicola Berretta ${ }^{2} \cdot$ Emerald Perlas $^{4}$ - Stefano Puglisi-Allegra ${ }^{1,2} \cdot$ Rossella Ventura $^{1,2}$}

Correction to: Mol Neurobiol. (2018) 55:7401-7412 https://doi.org/10.1007/s12035-018-0925-z

The original version of this article unfortunately contained a mistake in Figure 3. The drawing superimposed on photomicrographs to identify the region of Dorsal raphè Nuclei was inappropriately positioned. The corrected figure is given below. This correction does not change the value of results and the text of the figure legend, results, discussion, and conclusion. We apologize for the inconvenience.
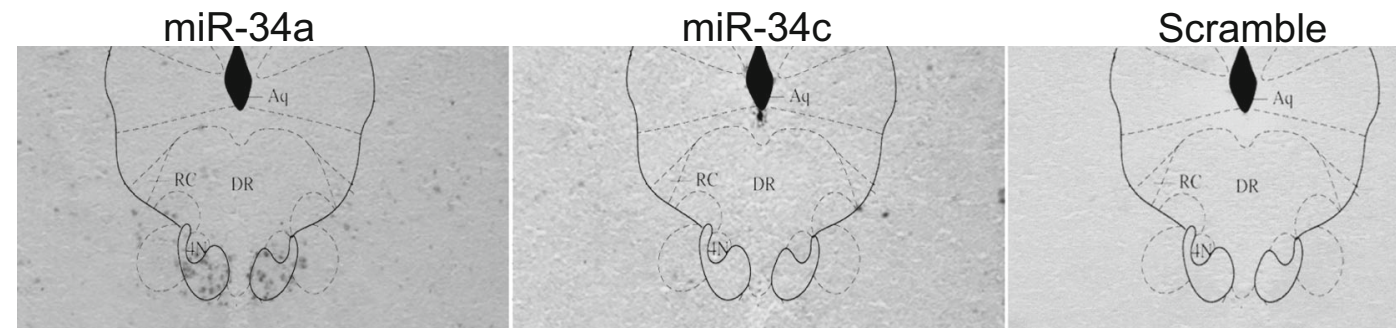

The online version of the original article can be found at https://doi.org/ 10.1007/s12035-018-0925-z

Diego Andolina

diego.andolina@uniroma1.it

1 Department of Psychology and Daniel Bovet Center, La Sapienza University, Piazzale Aldo Moro 5, 00181 Rome, Italy

2 Santa Lucia Foundation, European Center for Brain Research, Via del Fosso di Fiorano 64, 00143 Rome, Italy

3 CNR -Institute of Cell Biology and Neurobiology, Rome, Italy

4 Mouse Biology Unit, European Molecular Biology Laboratory (EMBL), Monterotondo Scalo, Rome, Italy 